\title{
Cotidianidad y significación: aproximaciones al tema de la memoria desde el pensamiento de Humberto Giannini*
}

\section{Cotidianidade e significação: abordagens do tema da memória a partir do pensamento de Humberto Giannini}

\section{Dailiness and Meaning: Approaches to the Memory Issue from Humberto Giannini’s Perspective}

\author{
Nelson VERGARA Muñoz ${ }^{* *}$
}

\begin{abstract}
RESUMEN
El trabajo reseña conceptos fundamentales de la Teoría de la Vida Cotidiana del filósofo chileno Humberto Giannini, con vistas a acercarla, desde dos nociones claves de la temporalidad, el tiempo ferial y el tiempo festivo, a lo que puede reconocerse como función vital de la Memoria. El propósito final es contribuir, teórica y metodológicamente, con tareas de rescate de la memoria colectiva de grupos sociales insertos en la vida cultural y natural, en su cotidianidad. Concebimos aquí, tanto como en la investigación que nos sirve de base, a la vida cotidiana como el ambiente natural y sociohistórico, esencial y primario, en el que se desarrolla la existencia humana y asumimos su interpretación desde una perspectiva filosófica. En este contexto, pensamos la memoria como una construcción sociohistórica, y valoramos su función como re-creadora de mundos vividos o soñados, más que como reproductora natural de acontecimientos. En tal situación deberá ser evidente el componente imaginario que la constituye. De aquí la importancia de la narración como factor esencial de todo proceso de rememoración y que se recoge en los relatos sagrados y profanos que van dando cuenta del devenir de los tiempos, en todos los lugares y lenguas, más específicamente, en toda cultura y ambiente. Para dar razón de estas tesis, el trabajo se inicia con la caracterización de las instancias básicas de toda cotidianidad, sigue con la reflexión en torno al tiempo y sus narraciones para fundamentar la emergencia histórica de la memoria y culmina en reflexiones fundamentales de los tiempos laborales, los tiempos festivos y el papel de la memoria en estas temporalidades, lo que debe informar del carácter sociohistórico, cultural y ambiental de los procesos de rememoración y, eventualmente, de olvido.
\end{abstract}

Palabras clave: rutina; transgresión; temporalidad; memoria.

\footnotetext{
"En una versión más breve este trabajo fue presentado como ponencia al Seminario de Investigación Territorio, Memoria y Sustentabilidad, realizado en Osorno el 17 de Noviembre de 2010. Tanto el seminario como el artículo son productos del Proyecto Fondecyt $\mathrm{N}^{\circ} 1080665$, "Las sociedades litorales como sistema de prácticas y saberes tradicionales y científicos: la caleta como modelo de imaginarios pesqueros y dinámicas de uso y apropiación de recursos marinos".

${ }^{* *}$ Professor do Departamento de Humanidades y Arte y del Centro de Estudios Regionales de la Universidad de Los Lagos, Osorno, Chile. Email: nvergara@ ulagos.cl; nvergar@gmail.com
} 


\title{
RESUMO
}

O trabalho apresenta uma resenha dos conceitos fundamentais da Teoria da Vida Cotidiana do filósofo chileno Humberto Giannini, a fim de esclarecê-la, partindo das noções-chave de temporalidade, o tempo das feiras e o tempo festivo, desde as quais se pode reconhecer uma função vital da memória. O principal objetivo é contribuir, teórica e metodologicamente, para o resgate da memória coletiva de grupos sociais inseridos em sua cotidianidade, na vida cultural e natural. Concebemos como primordiais e essenciais, desde a pesquisa que nos serve de base, a vida cotidiana, o ambiente natural e sócio-histórico, nos quais se desenvolvem a existência humana e assumimos sua interpretação desde uma perspectiva filosófica. Nesse contexto, pensamos a memória como uma construção sócio-histórica e avaliamos sua função como recriadora de mundos vividos ou sonhados, mais do que uma reprodução natural de acontecimentos. Em tal situação, o componente imaginário se mostra com toda a evidência. Por isso, a importância da narrativa como fator essencial de todo processo de rememorização, recolhida pelos relatos sagrados e profanos que expressam o devir dos tempos, em todos os lugares e línguas, em toda cultura e todo ambiente. Para fundamentar estas teses, o trabalho apresenta uma caracterização dos elementos básicos de toda cotidianidade, continua com a reflexão sobre o tempo e suas narrativas, para fundamentar a emergência histórica da memória, e culmina com reflexões fundamentais sobre os tempos de trabalho, os tempos festivos e o papel da memória nessas temporalidades; essas abordagens informam sobre os caracteres sócio-histórico, cultural e ambiental dos processos de rememorização e, eventualmente, de esquecimento.

Palavras-chave: rotina; transgressão; temporalidade; memória.

\begin{abstract}
This work reviews fundamental concepts of the Daily Life Theory, from the Chilean philosopher Humberto Giannini, in order to cast light on it, and is based on two key notions of temporality - the time of fairs and the festive time - from where a vital function of Memory can be the final purpose is to contribute, theoretically and methodologically, with works of rescue of the collective memory of social groups inserted in the cultural and natural life in their dailiness. Here we perceive, as much as in the investigation that serves us as basis, the daily life as the essential and primary socio-historical and natural environment, in which human existence develops and we assume its interpretation from a philosophical perspective. In this context, we think the memory as a socio-historical construction and assess its function as re-creator of lived or dreamed worlds, more than a natural reproducer of events. In that situation the imaginary component that constitutes it must be evident. Therefore we notice the importance of the narration as an essential factor of every remembrance process that is gathered from sacred to profane tales, which express the becoming of times, in all places and languages, more specifically, in every culture and environment. To justify these theses, this work initiates with the characterization of the basic instances of every dailiness, followed by the reflection around time and its narrations to ground the historical emergency of the memory, and culminates with fundamental reflections of the working times, festive times and the role of memory of these temporalities. These approaches inform about the socio-historical, cultural and environmental character of the remembrance processes and, eventually, of oblivion.
\end{abstract}

Key-words: routine; transgression; temporality; memory.

del tiempo: de la significación y el sentido del tiempo. Es

Desde toda teoría social, es un hecho que la vida cotidiana se configura como experiencia colectiva, cuya condición fundamental es presentarse como una experiencia cierto que también forman parte de su estructura dimensiones espaciales y lingüísticas, pero ellas están claramente condicionadas por el acontecimiento del pasar, en cuanto pasar algo a alguien, a una comunidad, a una sociedad, a la 
vida socioambiental de un grupo humano en circunstancias siempre muy concretas, a las que conviene llamar históricas.

Recogiendo un decir habitual, el filósofo chileno Humberto Giannini ${ }^{1}$ dice que, en general, la gente se refiere a la cotidianidad afirmando que es aquello que pasa todos los días, y a veces entendiendo que es aquello que pasa cuando en realidad no pasa nada, se entiende nada nuevo; noción habitual que destaca de preferencia su reiteración y que, por lo mismo, el pensamiento popular caracteriza como algo anodino, banal e insignificante. Pero este decir es muy injusto dice Giannini porque, aunque parece válido que en cierto modo se refiere a aquello que acontece, que pasa como sin pena ni gloria, hay también, paradójicamente, la certeza de que en la vida cotidiana, al pasar, algo queda siempre, o va quedando, y que al quedar se integra, a lo menos como huella, en ese modo de ser transeúnte que es propio de lo humano. Es decir, que la cotidianidad deja marcas también, señales a veces profundas, imborrables, que convocan la Memoria o que la eluden por una necesidad psicológica de Olvido. Por esto es aconsejable poner atención en esta expresión habitual del pasar y sacar de ella sus múltiples significaciones iniciales ${ }^{2}$.

Dos son los modos básicos con que se manifiesta esta condición pasajera, esencialmente pasajera, de la vida cotidiana. Y aunque esto afecta a todo el trayecto cotidiano y sus momentos claves, ambos modos se sitúan de preferencia en un mismo territorio simbólico: la calle, lugar privilegiado del pasar, del acontecer diario en los espacios socioambientales vividos por las personas y los grupos humanos. El primero, y que ante todo salta a la vista, el más habitual, el más conocido en tanto se hace alusión a la cotidianidad, se refiere a la constancia y regularidad de las cosas. Y así esta calle, "por la que yo - como tantos otros - voy y vengo todos los días, en la que todos los días, a una hora consabida, vuelven a repartirse los periódicos, a barrerse las veredas, a / levantarse las cortinas de las tiendas, a instalarse los vendedores callejeros, a pasar el recolector municipal etc." (GIANNINI, 1999b, p. 20-21).
De tal modo que entonces cotidiano "parece ser justamente lo que pasa cuando no pasa nada. Nada nuevo, habría que agregar" (GIANNINI, 1999b, p. 21). Pasar es entonces pasar siempre de un modo prácticamente 'unívoco', de tal modo regular que no nos sorprende, y en esa misma medida no lo divisamos; somos en él, pero no nos percatamos de su constancia. "Lo cotidiano es algo así, dice Giannini, y entonces aparece -y entonces se nos desaparece - como el antimisterio por excelencia, como la más tosca y desabrida de las rutinas" (GIANNINI, 1999b, p. 21).

Pero el término 'pasar' tiene referencias ambiguas que aquí resultan decisivas. Así, un segundo sentido, fundamental, surge, precisamente, con los rasgos más opuestos y, sin embargo, igualmente reales. Porque 'pasar' no solamente significa lo fluyente regular, lo que en su transitoriedad reiterad del mismo modo no deja huellas, al menos visibles, sino que también significa para nosotros "lo que repentinamente se instala en medio de la vida, lo que irrumpe en ella como novedad" (GIANNINI, 1999b, p. 21) y cambio. Ambos modos de ser pasajero, asegura Giannini, son igualmente decisivos en lo cotidiano y no puede sin más situarse la cotidianidad en uno o en otro, sino que en ambos. Rutina y Transgresión serán los nombres con que el pensador chileno identificará estas dos modalidades del pasar en el que desde ya o desde siempre estamos situados. Al menos formalmente. Y esta doble coordenada deberá manifestarse en todas las estructuras (territoriales, espaciales o socioambientales, temporales y lingüísticas) de la vida o experiencia común a la que nos acercamos arqueológicamente.

Topografía, Cronología y Expresión en algún lenguaje son tres caracteres esenciales de la estructura y significación de la cotidianidad. Lo que tienen de común estas dimensiones de lo cotidiano es que en ellas la experiencia común adquiere el ritmo y la figura circular, de inicio y retorno, de progreso y regreso que le es consustancial, que Giannini llama "reflexivo" y que comprometen su ser localizado, temporal y lingüístico ${ }^{3}$.

\footnotetext{
${ }^{1}$ Humberto Giannini ha sido profesor de Filosofía de la Universidad de Chile, conferenciante y escritor. Entre sus libros más representativos se encuentran $L a$ "reflexión" cotidiana, La experiencia moral, El mito de la autenticidad, La metafísica eres tú, así como gran cantidad de artículos publicados en revistas de Chile y del extranjero. Su línea fundamental de trabajo en estos últimos treinta años ha sido la investigación de la vida cotidiana desde una perspectiva que podemos llamar existencial, destacando en ella la dimensión moral. Cercano a Enrico Castelli y Paul Ricoeur, su trabajo reconoce deudas con pensadores como Platón, Aristóteles, Kant, en lo clásico, de Husserl y Heidegger en lo contemporáneo, pero siempre en una postura crítica. Actualmente es considerado como una de las figuras señeras en el cultivo del pensar filosófico de Chile y de Latinoamérica.

${ }^{2}$ En la investigación aludida hemos destacado esta dimensión cotidiana de la vida, expresándola como "prácticas cotidianas" que se desenvuelven en el tiempo. Cfr al respecto el sitio web de la investigación http://www.sociodiversity.org

${ }^{3}$ En una precisión que vale la pena destacar para no confundir el ciclo cotidiano con una suerte de circulo vicioso, H. Giannini dice que la vida cotidiana se manifiesta siempre como un modo de ser de alguien (una persona, una comunidad, una institución) que "viviendo se reitera silenciosamente y día a día ahonda en si mismo" (GIANNINI, 1999b, p. 19).
} 
Pero esta condición reflexiva no es unívoca: a lo menos posee tres sentidos que funcionan ligados entre sí formando una unidad compleja, férrea, aunque no invariable en la existencia social humana. Uno de ellos señala lo cotidiano en términos fundamentalmente espaciales: identifica los ámbitos por donde se desplaza la experiencia a partir de un ambiente o sitio al que se regresa diariamente, por lo que según Giannini adquiere el estatus de eje de todo el proceso. Este eje está representado por el domicilio. Otro es el sentido de la reflexión psíquica, esto es, la de una conciencia que se proyecta fuera de sí y que luego vuelve sobre sus propios contenidos para interrogarlos y hacerlos transparentes, en un movimiento análogo a las salidas y regresos del ritmo señalado en el primer caso. El tercero es el de la reflexión social que ya no ocurre esencialmente con relación al espacio sino al tiempo y que no la cumple el individuo sino que lo hace la sociedad histórica a la que el individuo pertenece. Este es particularmente el ámbito que nos interesa aquí y al que se incorpora el lenguaje como realidad sociohistórica. Tal reflexión tiene que ver con la recuperación que una sociedad histórica hace de su experiencia pasada (de su memoria, de su historia, de su apropiación del mundo natural con sus significaciones imaginarias y sensibilidad artística, de sus ceremonias y fiestas etc.) y se relaciona con lo que se ha llamado "traspaso de mundo" de una generación a otra en los cuales, incluso, se pasan y traspasan generacionalmente los saberes eco-productivos y simbólicos de los grupos humanos, así como las formas de cognición y percepción de la naturaleza y de la sociedad; es, en fin, el modo mediante el cual un conglomerado humano se reconoce como una sociedad que se relaciona y representa el mundo natural y social, con identidad propia ${ }^{4}$.

Ahora bien, Giannini sostiene que estos tres sentidos, al destacar el momento de su punto de partida y de regreso, lo que hacen es manifestar inequívocamente que el pasar cotidiano no es una línea que consista en pura pérdida de ser, sino que precisamente es también un retener, un parar el paso, en cierto modo un detenerlo aunque sea momentánea y a veces no tan momentáneamente, así como en otras ocasiones consista en lo contrario, en apurarlo, en romper por aceleración el ritmo habitual que tienen las cosas. De este modo, permanencia y cambio se instalan como coordenadas ineludibles en la configuración de lo cotidiano en cualquier espacio socioambiental. Así la retención actuará como un contratiempo cuya función será atesorar lo memorable en los recuerdos y en las cosas, con lo que nos atrincheramos en la vida y protegemos nuestra identidad. Y así es como la memoria se nos aparece en tanto momento generado por la experiencia cotidiana, fundamentalmente en el orden del tiempo evitando que el pasado, como dimensión fundamental de lo humano, se desvanezca sin dejar rastros. Más que un hecho natural, la memoria se nos presenta entonces como una construcción social y, como tal, está sostenida en y por el lenguaje, principalmente por la narración de los hechos y de los tiempos.

\section{2}

Pero, ¿qué es lo que atesora la memoria en esta experiencia de todos los días? Uno se sentiría tentado a identificar lo memorable con lo altamente significativo e importante, digamos con lo que, en el decir de Giannini, hace noticia. Sin embargo, el planteamiento que justifica la intención "arqueológica" ${ }^{5}$ del pensador chileno, es que este método arqueológico debe abrir camino hacia el sentido de algunos de los aspectos más banales de la vida diaria. Y esto, bajo el supuesto fundamental de que lo banal posee siempre un fondo significativo que actúa como sedimento de experiencias colectivas sepultadas o remotas, que operan al amparo de la conciencia inadvertida y echan sus raíces hasta el fondo mismo de la experiencia. El conocimiento de tales hechos, dice Giannini, podría encaminarnos hacia una conexión de sentido, sumergido, objetivado, "en las estructuras ya desleídas de los hechos", tal como en su momento lo sospechara S. Freud al someter a análisis fenómenos aparentemente anodinos e insignificantes como los sueños y aquello que con claridad meridiana y sentido de la oportunidad llamara "psicopatología de la vida cotidiana", fenómenos sin significado práctico tal vez, pero profundamente cargados de sentidos teóricos, es decir explicativo-interpretativos, tal como lo señalan las investigaciones de Norman Brown, Herbert Marcuse, Paul Ricoeur y Cornelius Castoriadis, entre otros.

\footnotetext{
${ }^{4}$ Cfr. Humberto Giannini, La metafísica eres tú.

5 "Así emplearemos el término tanto en el sentido etimológico: método que da razón de los fundamentos, de los principios; como en un sentido simbólico-histórico: la vía que conduce a cosas soterradas en el tiempo, invisibles para una conciencia" (1999b, p. 15), se entiende, para una conciencia personal, por cuanto, como se ha indicado, "ésta es visibilidad, 'presencia ante los sentidos' o clara evocación de dicha presencia” (1999b, p. 15).
} 
Pero, para estos efectos, no todo método es adecuado, aun cuando provengan de ciencias particulares habituadas al trato con las profundidades. Y esto es así porque la pregunta que intentamos hacer respecto de la conexión de sentidos o del sentido mismo de algo, "va más allá [trasciende] del cómo y del cuánto propios de una ciencia particular" (GIANNINI, 1999b, p. 43). Y como lo hemos venido señalando, agrega Giannini que

nuestra búsqueda quiere afinar su mirada en el terreno de lo culturalmente inadvertido, escudriñar entre esas cosas que se transfiere día a día de un sujeto a otro y que, no obstante, son permanentemente removidas, a causa de su carácter banal. Pero, eso que así se transfiere a espaldas de lo que hace noticia, puede incluso ser un signo de que allí hay justamente algo por investigar (1999b, 44).

Para la comprensión de esto nos detendremos en dos momentos temporales del proyecto/trayecto cotidiano: el tiempo del trabajo y el tiempo del reposo. Y esto, precisamente, porque de algún modo, trabajo y reposo representan los polos de la temporalidad humana, la que se hunde en lo más primordial y acaso sagrado de los tiempos ${ }^{6}$. Rescatar esas temporalidades, hacerlas presente de nuevo, traerlas a la memoria y mantenerlas en ella, es la tarea de la narración, signo inequívoco de la transgresión de la identidad, de $l o$ mismo, y advenimiento de lo fluyente, de lo que pasa, de $l o$ otro. Se narra lo que pasa, dice Giannini y que, "justamente por pasar no queda; salvo en la palabra que lo narra, salvo en la palabra del narrador que lo restituye a la realidad tal vez para iluminar ésta en su ser pasajero, tal vez por pura diversión" (GIANNINI, 1999b, p. 78).

Con otras palabras, afirma el pensador chileno, la narración lo es de algo que adviene, o más bien que irrumpe desde el no ser y se instala en medio de lo que tranquilamente está ahí, para presentarse como novedad y que lo hace digno de ser narrado.

Y este es el punto que ahora nos importa: narrar es transgredir el ser justamente en el órgano por el cual mantiene su virtud de ser: la identidad, es decir, el irremediable regreso a sí mismo, sin novedad ni historia. ¿Pero, no era este ser lo que definía la rutina? Por el contrario, lo que pasa, justamente pasando cambia el rumbo y el destino de aquello que, sometido a una ley o rutina, pasaba siempre: la dialéctica del pasar/quedar, de la que hemos hablado en otro lugar. Una transgresión, puesto que invierte la prioridad del ser sobre el devenir, de lo necesario-universal sobre lo contingente-individual; de lo rutinario sobre la novedad; y en vez de hacer derivar ésta, pacífica, mecánicamente, de aquel Ser universal, da vuelta la jerarquía y funda el ser o el hábito de ser de los entes en el Advenimiento (adventus) o en la irrupción de lo que Pasa: en la trizadura de lo continuo, de lo racional puro. Y es esta irrupción del triunfo siempre renovado y siempre inestable del mito sobre el logos (1999b, p. 79-80).

Transgresión, narración, historia: he aquí entonces los fundamentos de la necesidad de acordar y recordar los tiempos, la antinatural disposición favorecedora del cambio, de la búsqueda, que requiere de la memoria y que la va creando socialmente.

\section{3}

El tiempo civil, esto es, el tiempo de relojes y calendarios cuya pretensión es programar y regular ocios y negocios, ha sido obra, dice Giannini, de un largo proceso de ajustes con miras a concordar la divisibilidad de las mediciones temporales y hacer con-mensurables también este tiempo con la temporalidad cósmica total. En este proceso, continúa el filósofo, observamos inicialmente un origen y un desarrollo en el mito; posteriormente se ha querido, sin aniquilar su sentido primigéneo, pensar ese sentido de otros modos, pero sin abandonar su exigencia explicativa, la que en último término puede reducirse a hacer transitable el camino que va desde el tiempo desértico de la rutina hasta una trascendencia que lo cuenta sensatamente, situado al principio en los grandes ciclos naturales.

En otros términos: se ha buscado acordar esta temporalidad que fluye y se destruye -la temporalidad lineal y dispersiva de la historia humana - con aquella otra - la cósmica-, que 'avanza' continuamente hacia el principio y que progresando-regresando sostiene ante nuestra vista la figura sensible de 'lo mismo' y de lo eterno. Acordar la una es, platónicamente, el principio, la condición, para recordar la otra (1999b, p. 44).

${ }^{6} \mathrm{Cfr}$. Mircea Eliade, Mito y realidad y El mito del eterno retorno. 
Sin embargo, y aquí aparece lo que ahora nos interesa, no bastarán estos grandes ciclos de la naturaleza para aferrar el sentido propio de la temporalidad humana: hay que contar también las pequeñas historias de todos los días y ponerlas a salvo de aquella temporalidad genérica o de ese tiempo vacío y homogéneo de la temporalidad pura de los relojes y calendarios. De esta manera, el tiempo puede aparecérsenos como tiempo vivido que nos protege de ese otro tiempo de puras postergaciones que la mundanización va generando, o como tiempo del acuerdo que nos pone a salvo del olvido de nuestro ser, o como tiempo reflexivo que nos asegura el reencuentro con nosotros mismos.

Esta des-mundanización del tiempo, su alejamiento de la red de tramitaciones en que consiste el mundo y en el que siempre estamos atenidos a ganar tiempo, postula entonces, a veces muy oscuramente, una reflexión en la que se unifican los tiempos fragmentados y dispersos de la rutina y sus tiempos feriales, señalando hacia otro tiempo, el tiempo festivo en que se constituye e instituye no la competencia, sino la com-patencia y el re-encuentro: compatencia de un tiempo que quisiéramos salvar del olvido, de la dispersión y el anonimato, compatencia al fin de lo aparentemente banal e insignificante, pero en quienes se descubren dignidades suficientes que exigen ser contadas, narradas, salvadas de un trayecto sin retorno y por lo mismo sin recuerdo.

Entonces el tiempo del mito otra vez con sus sentidos profundos: el de acordar los tiempos con el fin de recordarlos, y el de recordar los tiempos con el fin de orientar la existencia. Desde ella podemos confirmar entonces que la memoria no es una instancia natural, espontánea, rutinaria, que pertenezca al hombre desde siempre como una mera facultad donada o regalada, sino que pertenece precisamente a su negación: así, la memoria no es, por tanto, una simple reproducción o representación de mundos, sino las múltiples y diversas re-construcciones imaginarias de sus sentidos.

Disponibilidad para el otro a fin de ser para sí y disponibilidad para sí mismo, son los caracteres que H. Giannini destaca como esenciales en la determinación del tiempo laboral y del tiempo festivo respectivamente. El primero se nos aparece notoriamente ligado a las normas que lo hacen rodar, a lo desde ya previsto, a lo consabido, a lo que hay que hacer para conservar el trabajo, esto es, a lo que se ha llamado la rutina de lo cotidiano. Pertenece al mundo de relojes y calendarios, pero fundamentalmente al mundo de la tramitación que pueda asegurar los propósitos y fines que un grupo humano se ha agenciado para proyectar su futuro. Es el mundo que, ganado con la voluntad y dedicación suficiente, puede hacer posible que fructifique el abandono de la seguridad domiciliaria, del nido, de las cosas conformadas para los requerimientos del sujeto. Mundo objetivado y externalizado, común, abierto en torno del otro y cerrado también en torno de las necesidades e intereses del otro, con quienes establecemos relaciones de jerarquías, muchas veces oscuras o invisibles. Entonces, si bien solemos reconocer al otro por alguna marca, no lo conocemos efectiva y claramente. $Y$ entonces, bajo estas condiciones nuestras representaciones imaginarias, particularmente la memoria, son un mecanismo que sacrifica creación por certeza, invento por seguridad, cerrando al máximo los ángulos del riesgo, del riesgo de perder el trabajo y quedarnos, como dice Giannini, literalmente, "en la calle". En este sentido representa el momento más funcional de la memoria. Las cosas, las personas, las instituciones adquieren allí la significación de lo que necesita seguir siendo lo que es, sólo con las variaciones suficientes para reconocer lo mismo en lo Mismo y asegurar el regreso a casa con la convicción de que esas cosas, personas, instituciones, nos estarán esperando mañana, requiriendo de nosotros solamente los caracteres funcionales de la eficiencia o eficacia, o como se ha dicho más arriba, de la competencia. Hay que recordar lo que se debe recordar, a fin de mantener y en lo posible acrecentar la competencia y de este modo asegurar el triunfo en una carrera desplegada con vistas a incentivos también funcionales $\mathrm{y}$, a veces, proporcionales al esfuerzo que se nos exige desde las perspectivas del Otro.

Y cabe afirmar que el grado de distancia y exterioridad de este ser para otro, respecto de un ser para sí, en otro tiempo y en otro lugar, define con bastante exactitud el tipo de sociedad a que se pertenece: desde la sociedad en la que el trabajo vende, enajena sus fuerzas simplemente a cambio de una paga - la ignominia del trabajo como mercancía - hasta la sociedad en que el trabajo implica esencial y concretamente un ser para nosotros, esto es, una efectiva comunidad en la inteligencia y en el destino de la obra. El primer signo de un tiempo democrático común (GIANNINI, 1999b, p. 27).

Como se ha señalado, estos modos de ser van acompañados por modos de comunicación. En el trabajo se ela- 
boran así dos modos que tienen como objeto el mismo afán ecoproductivo y funcional: el vertical, jerarquizado (el jefe, la clientela, el otro tiene la razón) y el horizontal competitivo, agonal (cada cual ha de dar lo mejor de sí para aventajar a los otros en el escalafón funcionario...) "Visto así, desde un trabajo mediatizado, el mundo representa un sistema de signos, de "valores intercambiables", de ofertas y postergaciones. El mundo es feria" (GIANNINI, 1999b, p. 28).

En el libro La experiencia moral, Giannini agrega algunas precisiones que vale la pena tener presente. En primer lugar, la noción misma del trabajo se presenta ahora cualificada como del ser-valor del mundo y no mera apropiación del mundo o de la naturaleza. En segundo lugar, esta apropiación tiene un sentido productivo y no simplemente comprensivo. Así, se comprende el mundo para obtener de alguna manera un producto, una obra que está hecha en vista de lo Otro y de los Otros. De este modo, el trabajo "es el espacio de la exposición a lo Otro, y de la disponibilidad para los Otros" y sus exigencias (GIANNINI, 1992, p. 21).

En este espacio, sostiene el filósofo, la disponibilidad para los otros más que acoger, "coge" sólo aquello que se encuentra en la vía de nuestros fines inmediatos; y lo cogerá sólo en tanto y durante el tiempo en que permanece en la línea de tales fines... A la postre - y esto es clave - pertenece al tiempo de una falsa disponibilidad, o dicho de otro modo, a una falsa disponibilidad de tiempo (GIANNINI, 1992, p. 22).

Hay razones... para considerar el trabajo como el topos de la alienación, en el sentido que la etimología de la palabra nos señala. El trabajoes, en efecto, el foco hacia el cual se vuelve sistemáticamente el ser domiciliado (ensimismado) para apropiarse de una cualidad delmundo. Y es alienación en grado máximo y en esa acepción negativa que le ha dado el marxismo, cuando se lo considera sólo como una mercancía subordinada, como todas, a "la ley natural" de la oferta y la demanda. Pero lo es también cuando la disponibilidad para los otros está a todas luces mediatizada por la espera de una disponibilidad para sí mismo - en otro lugar y en otro tiempo - olvidada en el camino (GIANNINI, 1992, p. 22).

En tal situación, la alienación en el trabajo es, a la par, la enajenación de la memoria, su captura por lo funcional $\mathrm{y}$ funcionario.

Sin embargo, lo referido no constituye un destino, una fatalidad. El mundo, si bien es feria, no lo es siempre.
Hay también lo festivo, el festival, la fiesta, el regreso a sí mismo, al tiempo de la disponibilidad del sujeto (individuo, comunidad, sociedad) para sí mismo, en un espacio y tiempo en que el hombre se protege de lo extraño, del peligro de lo extraño, o como decía Ortega, del peligro que es el Otro. Tal regreso a sí mismo es posible por cuanto entramos en un espacio conformado por tiempos y lenguajes, cosas familiares que nos son disponibles en un orden que está vuelto hacia nosotros.

Pero hay algo más: el domicilio, a causa de esta función 'reflexiva` que la constituye ha de ser la clave insustituible que me permite aventurarme más allá, hacia el mundo, en el proyecto cotidiano de ganarme la vida y regresar luego a él, desde cualquier horizonte, como a lo más propio (GIANNINI, 1999b, p. 25).

En La experiencia moral dirá Giannini lo que nos resulta esencial para lo que estamos refiriendo: que el regreso a sí mismo no es una retirada del mundo en el sentido de una huída de él, un evitarlo, sino más bien lo contrario, la posibilidad de acceder transgresivamente a su mejoramiento, a la posibilidad de no aceptar lo que meramente hay, sino que fundamentalmente hacer ser, que advenga, lo que ahí no hay, a su creación constante. De este modo,

el domicilio, en función de centro re-flexivo, es la clave insustituible que me permite aventurarme más allá, hacia lo desconocido, lo nuevo, lo imaginario, lo extraño, lo inquietante... hacia lo Otro, para reencontrar luego, la "realidad" y reintegrarme a ella. Dicho platónicamente: a condición de no olvidar jamás el secreto del retorno [a lo propio] (GIANNINI, 1992, p. 18).

Y para esto, los signos que suscitan la memoria ya no son el pasado, la historia sida, la tradición, sino el cambio, la innovación, la imaginación creadora de mundos que no están ahí, pero que pueden estarlo y ser compatibles con el azar, la incertidumbre, la complejidad, el desorden, el caos en cuanto realidades insoslayables y perentorias. Estos nuevos signos que motivan recordar, son signos también de una nueva comprensión de la memoria, así como de nuevos sistemas de significaciones que se apoderan hoy día de nuestros mundos cotidianos en distintos espacios socioambientales. Por tanto, el tiempo es fiesta también, carnaval, mitología. He aquí las nuevas realidades. Pero, 
he aquí también los nuevos desafíos... y peligros, ya que perfectamente, la fiesta, el carnaval, la mitología puede transformársenos en mercancías - incluso la propia natura-

\section{Bibliografía}

BROWN, Norman. Eros y Tanatos. México: Joaquin Mortiz, 1972.

CASTORIADIS, Cornelius. La institución imaginaria de la sociedad. Buenos Aires: Tusquets, 2007.

ELIADE, Mircea. Mito y realidad. Madrid: Guadarrama, 1968. 2003. El mito del eterno retorno. Madrid: Alianza Editorial,

FREUD, Sigmund. Obras completas. v. 1. Madrid: Biblioteca Nueva, 1948.

GIANNINI, Humberto. Desde las palabras. Santiago de Chile: Nueva Universidad. Universidad Católica de Chile, 1981. taria, 1992.

La experiencia moral. Santiago de Chile: Universi. Metafisica del lenguaje. Santiago de Chile: LOM

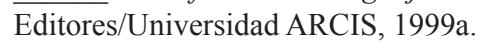

La "reflexión" cotidiana. Hacia una arqueología de la experiencia. Santiago de Chile: Universitaria, 1999 b. leza. Y para esto hay también bastantes signos en nuestros tiempos.

. El pasar del tiempo y su medida. Santiago de Chile: Universitaria, 2001.

. La Metafisica eres tú. Una reflexión ética sobre la intersubjetividad. Santiago de Chile: Catalonia, 2007.

MARCUSE, Herbert. Eros y civilización. Barcelona: Ariel, 2003.

ORTEGA Y GASSET, José. El hombre y la gente. Madrid, Revista de Occidente, tomo II,1962,. (Col. El Arquero), 212 páginas.

RICOEUR, Paul. Historia y narratividad. Barcelona: Paidós Ibérica, 1999.

THER, F.; GAJARDO, G.; VERGARA, N. "Fundamentación teórica" del proyecto de investigación 1080665 financiado por Fondecyt, Chile, 2008-2010.

VERGARA M., Nelson. "Saberes y entornos. Notas para una epistemología del territorio". Revista ALPHA, Universidad de Los Lagos-Osorno, n. 31, p. 163-174, 2010.

Recebido em março de 2011.

Aceito em abril de 2011.

Publicado em junho de 2011. 